\title{
Marcas de calidad de carne de ovino y caprino ¿ Un hecho o una ficción?
}

\author{
Teixeira, A.
}

ESA- Instituto Politécnico de Bragança. Centro de Ciência Animal e Veterinária (CECAV). Campus de Sta. Apolónia. Bragança. Portugal.

\section{DOP. \\ IGP. \\ Autenticidad. \\ Carne. \\ Ovino. \\ Caprino.}

PALABRAS CLAVE ADICIONALES

\section{ADDITIONAL KEYWORDS}

POD.

PGI.

\section{Authenticity.}

Meat.

Sheep.

Goat.

\section{INFORMACIÓN}

Cronología del artículo.

Recibido/received: 10.05 .15

Aceptado/Accept: 20.03.16

Online: 15.09 .16

Correspondencia a los autores/Contact e-mail:

teixeira@ipb.pt

\section{RESUMEN}

\begin{abstract}
Se hace un análisis crítico de la importancia de las marcas de calidad de carne de ovino y caprino (DOP e IGP) en Portugal y España como un elemento diferenciador en un mercado globalizado, una forma de dinamizar la producción en áreas geográficas, algunas de ellas social y económicamente deprimidas, y como una manera de preservar los patrimonios genético y cultural. Se discute la necesidad de encontrar estrategias basadas en métodos analíticos que comprueben la individualidad, especificidad y garanticen la autenticidad de productos DOP e IGP, como un tipo de protección adicional basada en métodos objetivos comprobados científicamente.
\end{abstract}

\section{Quality labels of sheep and goats meat. A fact or a fiction?}

\section{SUMMARY}

A critical analysis of the importance of quality brands (PDO and PGI) of sheep and goat meat in Portugal and Spain as an element differentiator in a globalize market was made as a way to support the animal production, revitalize socially and economically depressed areas and to preserve a genetic and cultural patrimony. The need to find strategies based on analytical methods to check the individuality, the specificity was discussed as well to ensure the authenticity of products, as a kind of an extra protection based on scientifically checked methods.

\section{INTRODUCCIÓN}

La globalización ha dado lugar a un aumento de la competitividad, a la cual la producción de carne y de productos cárnicos no es una excepción, por ello se requiere un aumento de la eficiencia en la forma de producción, transformación y comercialización. Por otro lado, la globalización conlleva la necesidad de una producción en escala, lo que limita la accesibilidad a los mercados de los pequeños y medianos productores, que son excluidos de los principales circuitos comerciales. En España y Portugal, el camino iniciado ya hace algún tiempo, con la apuesta del sector cárnico a los sistemas de calidad diferenciada, ha propiciado un incremento de marcas de calidad con denominación de origen protegida, indicación geográfica protegida o de especialidad tradicional garantizad (DOP, IGP y ETG) registra- das por la Unión Europea. En el caso particular de los ovinos y caprinos, en la península Ibérica la gran mayoría de las marcas están asociadas a razas autóctonas y totalizan, en conjunto, 15 marcas DOP e IGP de ovinos y 5 de caprinos (figuras 1, 2 y 3). Estas marcas de calidad constituyen hoy un elemento diferenciador en el mercado, dinamizador de la producción con revitalización de áreas o regiones geográficas, que proporciona una mayor confianza al consumidor y ayuda a conquistar nuevos mercados, más allá de ser un apoyo para preservar un patrimonio cultural y genético importante.

\section{¿DOP E IGP, UN HECHO O UNA FICCIÓN?}

\section{UN HECHO:}

- Las marcas DOP e IGP de carne de ovino y caprino 
son una realidad que se debe, en gran medida, a la diversidad del patrimonio genético de los ovinos y caprinos en la Península Ibérica: 11 razas ovinas y 5 caprinas autóctonas de Portugal (AniDoP, 2015), 45 razas ovinas y 21 caprinas autóctonas de España (FEAGAS, 2015);

- La existencia de asociaciones de criadores que preservan y seleccionan estas razas, desde siempre, ha hecho que no se haya perdido este importante patrimonio genético, contribuyendo para que fuera posible, a través de sus agrupamientos de productores, solicitar a la Unión Europea (UE) que se les otorgara las marcas de calidad que hoy son reconocidas;

- El reconocimiento de la UE y de las administraciones locales (por intermedio de bases legales, reglamentos, despachos normativos, Reales-Decretos, publicados en diarios oficiales) así como el compromiso de los productores en la gestión de las marcas, aseguran su continuidad y la defensa de la reputación asociada a las mismas;

- La organización de cooperativas y asociaciones ha hecho que los productores se organicen para la defensa de sus marcas y comercialicen sus productos de calidad diferenciada frente a otros genéricos;

- La definición de un cuaderno de especificaciones define la individualidad y especificidad de estos productos frente a otros, con los cuales no deben ser confundidos. Factores como la raza, tipos de animal y canal producidas; características orográficas y climáticas; diversidad de los suelos; tipo de vegetación; sistemas de agricultura que condicionan la producción y forma de cría de los ovinos y caprinos, son la mayoría de las veces aspectos diferenciadores que generan la individualidad propia de los productos de una determinada región;

- Aspectos socio-culturales, principalmente los relacionados con el saber hacer de las gentes, con las tradiciones de consumo, y costumbres culinarias que pasaron de generación en generación, son factores diferenciadores de los productos con marca hoy reconocidos;

- La constitución de un organismo privado de control y certificación debe asegurar que todas las normas de producción, procesamiento, transporte y comercialización de los productos, cumplan estrictamente las normas de los respectivos cuadernos de especificación.

- La figura 4 esquematiza todas las relaciones entre las distintas organizaciones que promocionan la producción y comercialización de carnes con marcas DOP e IGP, debiendo notarse la importancia del carácter priva-

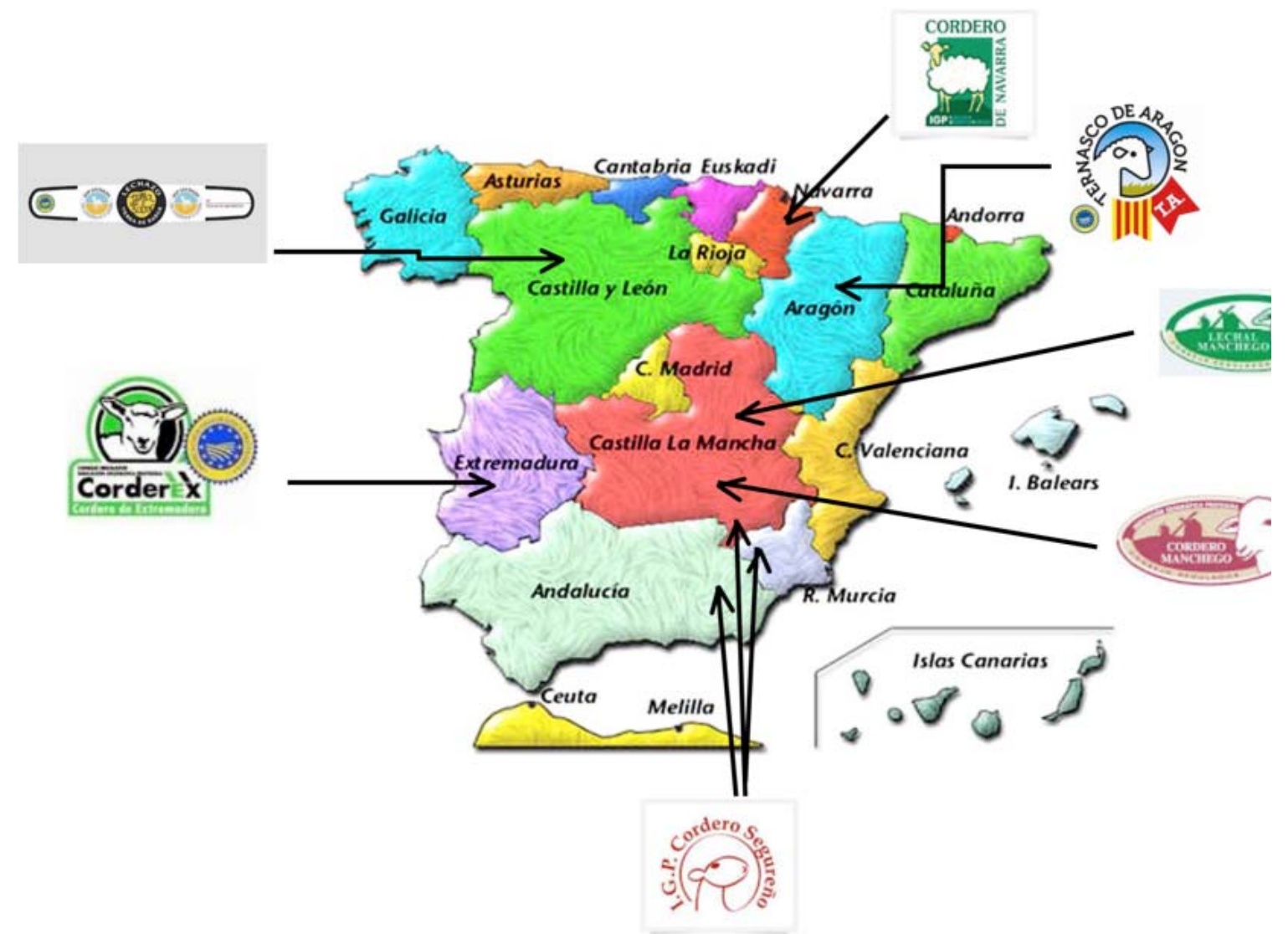

Figura 1. Áreas geográficas de producción de carne de ovino con IGP en España (Geographic areas of sheep meat production with PGI in Spain) (Fuente: Teixeira et al., 2014). 
do que los organismos privados de control y certificación deben poseer, cultivando la ausencia de cualquier relación de estabilidad con los demás intervinientes de la cadena, desde la producción a la comercialización.

\section{UNA FICCIÓN:}

- ¿Un sello o logo de certificación, colocado en la canal, garantiza la autenticidad de un producto con marca DOP o IGP?

Sí, principalmente por tratarse de un sello que es otorgado por la entidad certificadora de la marca DOP o IGP, esta entidad debe estar acreditada por una entidad nacional de acreditación, de acuerdo con la norma de Evaluación de la Conformidad, siguiendo los requisitos para organismos que certifican productos, procesos y servicios (UNE-EN ISO/IEC 17065/2012).

No, puesto que no hay absoluta seguridad que en todo momento de la cadena productiva el organismo certificador haya controlado todos los eslabones.

- ¿Es, en su acción, el organismo certificador independiente de todos los demás organismos de la cadena productiva?

No siempre es posible verificar una certeza absoluta de tal independencia. Ocurre que en muchas situaciones el organismo privado de control y certificación es el mismo para varias marcas y puede estar demasiado comprometido con las mismas. Sin embargo, con la norma UNE antes mencionada, el organismo de certificación debe garantizar en todo momento su independencia respecto a los auditados a los que se les certifica el producto.

- ¿Está el organismo certificador dotado de las herramientas que le permitan garantizar la autenticidad de un producto?
- Sede de Distrito

- Outras localidades Limite de Distrito Cordeiro do Barroso Borrego Serra da Estrela Borrego Terrincho Borrego de Montemor-o-Novo Borrego do Baixo Alentejo Borrego da Beira Cordeiro Bragançano Borrego do Nordeste Alentejano Canhono Mirandês

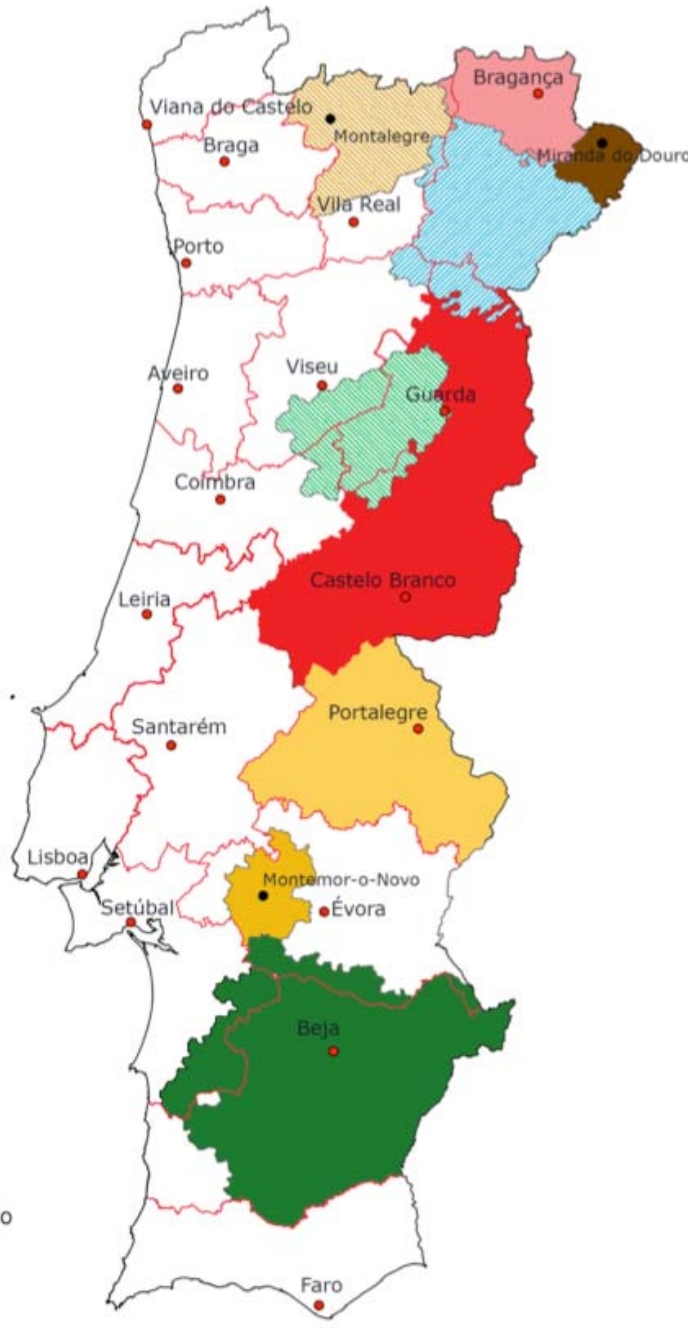

Figura 2. Áreas Geográficas de producción de carne de ovino en Portugal con DOP e IGP (Geographic areas of sheep meat production with DOP and PGI in Portugal) (Fuente: Teixeira et al., 2014).
No siempre. Por ejemplo, hay ocasiones en las que el organismo de control y certificación, en caso de sospecha de alguna irregularidad, no recurre a métodos analíticos objetivos.

- ¿Es la medida de la composición de la carne una garantía de autenticidad de un producto?

Es evidente que la composición varía considerablemente entre especies y razas, pero dentro de la misma especie o raza, e incluso dentro de la misma canal, ocurren variaciones en la composición. Aunque la carne sea definida como la porción comestible de la canal, la definición está abierta a varias interpretaciones, que se pueden relacionar con aspectos de calidad alimenticia, de aceptabilidad o incluso a cuestiones económicas. Por ejemplo, véase la diferencia entre las piezas pierna y costillar de una misma canal (Panea et al., 2012; Rodrigues et al., 2006; Teixeira et al., 2004 y 2005; Sañudo et al., 2000, 2012).

¡El análisis de la composición determinado por métodos químicos de referencia es muy utilizado en términos de caracterización de un producto cárnico! ¿Pero será suficiente para comprobar la autenticidad del producto? Los análisis químicos de los principales componentes de la carne pueden sufrir pequeñas variaciones que se pueden reflejar en el flavor, textura, color o, incluso, en su calidad nutritiva (Rodrigues y Teixeira, 2009, 2010, 2013; Horcada et al., 2012; Guerrero et al., 2015). Otro aspecto importante se relaciona con la presencia o 


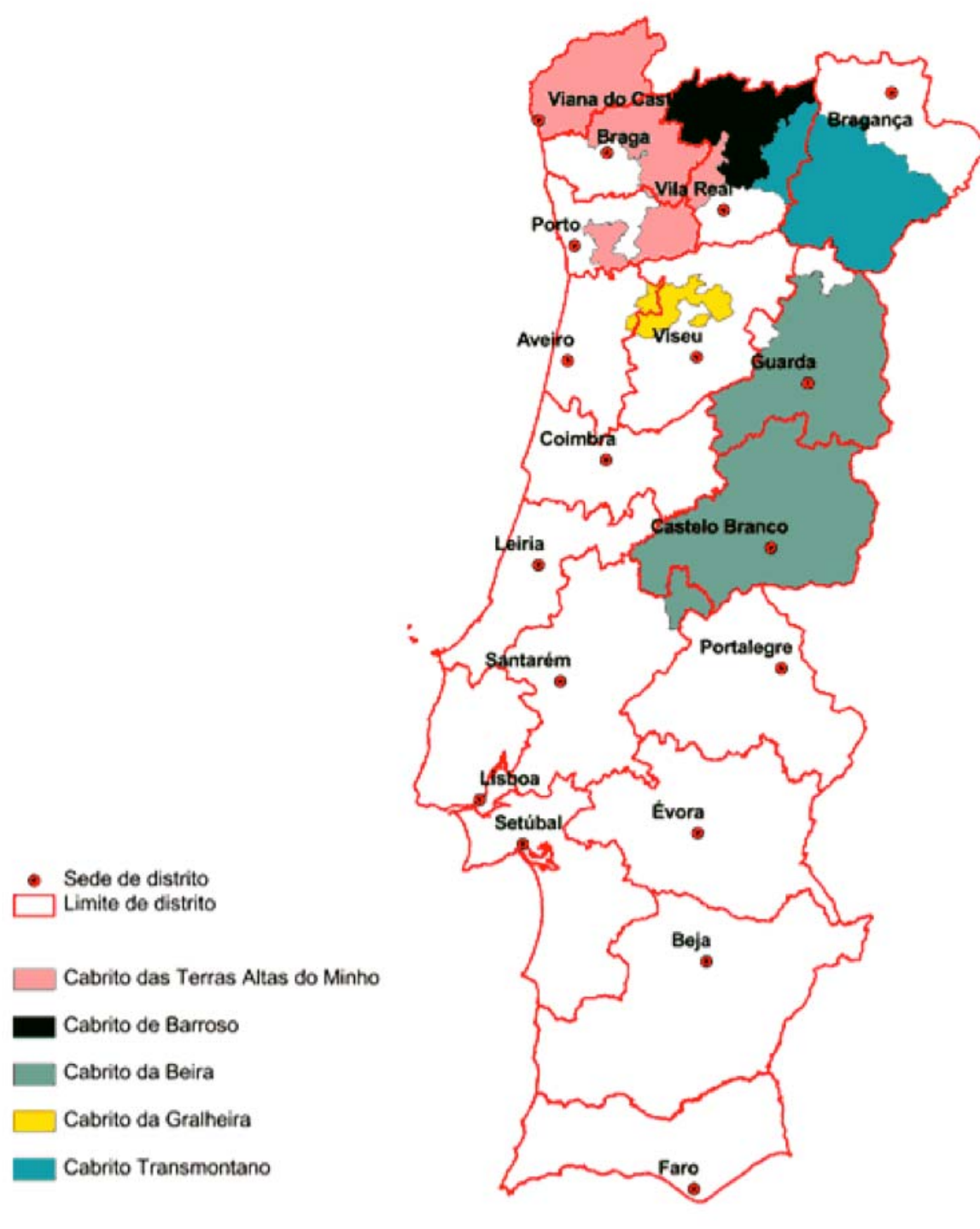

Figura 3. Áreas geográficas de producción de carne de caprino en Portugal con DOP e IGP (Geographic areas of goat meat production with DOP and PGI in Portugal). (Fuente: GPPAA, MADRP, 2009).

ausencia de ciertos compuestos traza que pueden encontrase tanto del músculo como de la fracción lipídica de la carne.

De todas las variaciones que pueden ser consideradas en la carne como desvíos de la autenticidad, las inherentes a diferencias debidas a la raza, sexo, edad o aspectos relacionados con el manejo (principalmente alimentario) son las que pueden repercutir en la composición del músculo o de las distintas fracciones de grasa. Unas pueden depender más de las características intrínsecas del propio animal y del sector productivo, mientras otras se relacionan más con las condiciones pre-sacrificio, sacrificio y post-sacrifico, que pueden igualmente provocar desvíos de calidad (Jiménez-Badillo et al., 2009; Teixeira et al., 2011; Ripoll et al., 2012). Ade- más de la calidad tecnológica de la carne, determinada por la medida del $\mathrm{pH}$, de la capacidad de retención de agua, actividad del agua, textura, color o hasta de análisis sensorial, es posible hoy en día utilizar otras técnicas: la inmunodifusión, inmunoelectroforesis o ensayos de inmunoabsorción enzimática (ELISAs) bien como métodos basados en la caracterización del DNA (PCR) y análisis de isótopos estables en esquemas de rastreabilidad y verificación de la autenticidad de carne y productos cárnicos (Ayaz et al., 2006; Ballin et al., 2009; Ballin, 2010; Ali et al., 2014), la discriminación de carnes por infrarrojo en campo cercano (NIRS) (Alomar et al., 2003), la autenticación de productos halal/kocher (Demirhan et al., 2012), la discriminación entre carnes frescas y descongeladas (Ballin y Lametsch, 2008) o bien la autenticación geográfica del origen de la carne (Franke, et al., 2008; Dalvit et al., 2008).

\section{CONCLUSIÓN}

El futuro de los productos DOP o IGP parece no estar sólo conectado a su promoción y protección internacional, sino también a las estrategias basadas en métodos analíticos que comprueben su individualidad, especificidad y garanticen su autenticidad, en el sentido de garantizar que un producto posee las características y proviene de las fuentes anunciadas, y de que no fue objeto de alteraciones a lo largo de un proceso productivo, significando que dicho producto fue producido, transportando y vendido de la forma que se corresponda a las expectativas asociadas a ese mismo producto.

Aunque la mayoría de los productores de DOP/IGP de carnes de ovino y caprino de la Península Ibérica utilizan su marca como un medio de protección o de marketing, es interesante observar que la gran mayoría no utilizan ningún tipo de protección adicional, basada en métodos objetivos y comprobados científicamente.

Es cierto que las carnes con marcas DOP/IGP ofrecen una mayor protección del nombre y la importancia de la certificación de terceros que otras carnes, lo cual es visto como una forma de reforzar la credibilidad del pro- 


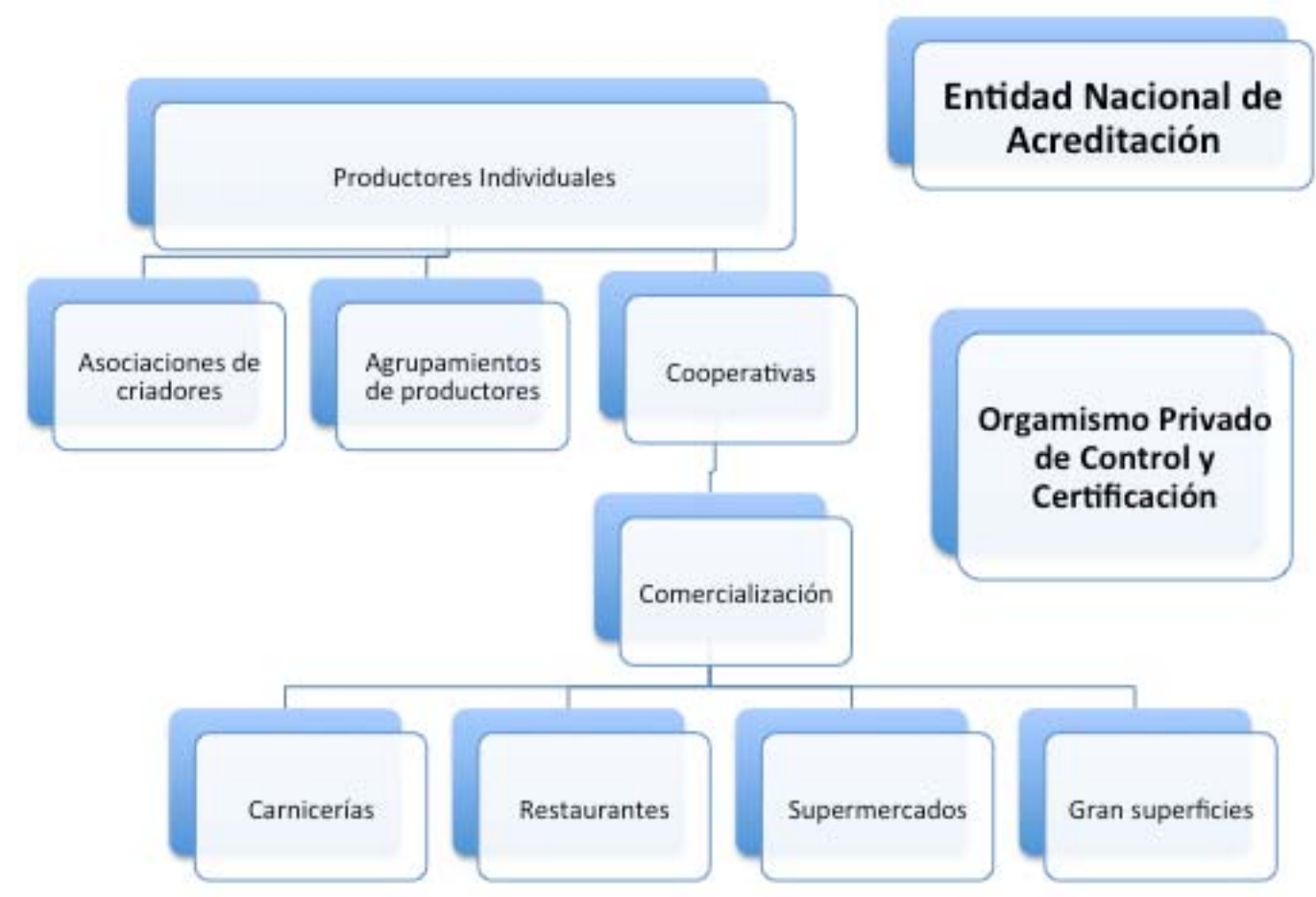

Figura 4. Representación esquemática de la organización de la producción y comercialización de productos con marcas DOP e IGP (Schematic representation of the organization of production and marketing of products with PDO and PGI brands).

ducto. Por otro lado, no siempre el consumidor es capaz de reconocer o entender estos instrumentos.

\section{¿Qué PASARÁ en El FUtURO?}

Si queremos que DOP/IGP sean entendidas como herramientas de marketing, y un compromiso de garantía de calidad y seguridad alimenticia, tenemos que tener en cuenta otros elementos que logren la máxima reducción de riesgos, aplicando el principio de prevención continua de la granja a la mesa, con el establecimiento de estrategias de control de base científica de máxima transparencia, y con la interacción entre todas las partes interesadas. En definitiva: No matando la gallina de los huevos de oro.

\section{BIBLIOGRAFÍA}

Ali, M.E.; Razzak, M.A. and Abd-Hamid, S.B. 2014. Multiplex PCR in species authentication: probability and prospects. A review. Food Anal Meth, 7: 1933-1949.

Alomar, D.; Gallo, C.; Castaneda, M. and Fuchlocher, R. 2003. Chemical and discriminant analysis of bovine meat by near infrared reflectance spectroscopy (NIRS). Meat Sci, 63: 441450.

AniDoP. 2015. Animais domésticos de Portugal. http://anidop.net/ index.php/racas-autoctones (10/02/2015).

Ayaz, Y.; Ayaz, N.D. and Erol, I. 2006. Detection of species in meat and meat products using enzyme-linked immunosorbent assay. J Muscle Foods 17: 214-220.

Ballin, N.Z. and Lametsch, R. 2008. Analytical methods for authentication of fresh vs. thawed meat. A review. Meat Sci, 80 : 151-158.

Ballin, N.Z.; Vongensen, F.K. and Karlsson, A.H. 2009. Species determination - Can we detect and and quantify meat adulteration? Meat Sci, 83: 165-174.

Ballin, N.Z. 2010. Authentication of meat and meat products. Meat Sci, 86: 577-587.

Dalvit, C.; De Marchi, M.; Dal Zotto, R.; Gervaso, M.; Meuwissen, T. and Cassandro, M. 2008. Breed assignment test in four Italian beef cattle breeds. Meat Sci, 80: 389-395.

Demirhan, Y.; Ulca, P. and Senyuva, H.Z. 2012. Detection of porcine DNA in gelatine and gelatine containing processed food products-Halal/Kosher authentication. Meat Sci, 90: 686-689.

Feagas. 2015. Catálogo Oficial de Razas de España. http:// feagas.com/index.php/es/razas/especie-ovina\#.VNTpxUIRTfg (10/02/2015).

Franke, B.M.; Haldimann, M.; Gremaud, G.; Bosset, J.O.; Hadorn, R. and Kreuzer, M. 2008. Element signature analysis: its validation as a tool for geographic authentication of the origin of dried beef and poultry meat. Europ Food Res Technol, 227: 701-708.

Gabinete de Planeamento e Políticas Agro Alimentar. Ministeìrio da Agricultura, Desenvolvimento Rural e Pescas. 2009. Anuário Pecuário (Animal Production Yearbook) 2008-09.

Guerrero, A.; Campo, M.M.; Olleta, J.L.; Resconi, V.C.; Muela, E. y Sañudo, C. 2015. Estudio comparativo de la calidad de la canal y la carne de lechales de raza Churra Tensina y dos tipos ovinos comerciales amparados bajo marcas de calidad. Arch Zootec, 64: 211-220.

Horcada, A.; Ripoll, G.; Alcade, M.J.; Sañudo, C.; Teixeira, A. and Panea, B. 2012. Fatty acid profile of three adipose depots in 
seven Spanish breeds of suckling kids. Meat Sci, 92: 89-96.

Jiménez-Badillo, M.R.; Rodrigues, S.; Sañudo, C. and Teixeira, A. 2009. Non-genetic factors affecting live weight and daily gain weight in Serrana Transmontano kids. Small Rum Res, 84: 125-128.

Panea, B.; Ripoll, G.; Horcada, A.; Sañudo, C.; Teixeira, A. and Alcade, M.J. 2012. Influence of breed, milk diet and slaughter weight on carcass traits of suckling kids from seven Spanish breeds. Spanish J Agric Res, 10: 1025-1036.

Ripoll, G.; Alcade, M.J.; Horcada, A.; Sañudo, C.; Teixeira, A. and Panea, B. 2012. Effect of slaughter weight and breed on instrumental and sensory meat quality of suckling kids. Meat Sci, 92: 62-70.

Rodrigues, S.; Cadavez, V. and Teixeira, A. 2006. Breed and maturity effects on Churra Galega Bragançana and Suffolk lamb carcass characteristics: killing-out proportion and composition. Meat Sci, 72: 288-293.

Rodrigues, S. and Teixeira, A. 2009. Effect of sex and carcass weight on sensory quality of goat meat of Cabrito Transmontana. J Anim Sci, 87: 711-715.

Rodrigues, S. and Teixeira, A. 2010. Consumers' preferences for meat of Cabrito Transmontano. Effects of sex and carcass weight. Spanish J Agric Res, 8: 936-945.

Rodrigues, S. and Teixeira, A. 2013. Use of generalized procrustes analysis (GPA) to test the effects of sex and carcass weight on sensroy quality evaluations of Terrincho lamb meat. Meat Sci, 93: $485-488$.
Sañudo, C.; Alfonso, M.; Sánchez, A.; Delfa, R. and Teixeira, A. 2000. Carcass and meat quality in light lambs from different fat classes in the EU carcass classification system. Meat Sci, 56: 8994.

Sañudo, C.; Campo, M.M.; Muela, E.; Olleta, J.L.; Delfa, R.; JiménezBadillo, R.; Alcalde, M.J.; Horcada, A.; Oliveira, I. and Cilla, I. 2012. Carcass characteristics and instrumental meat quality of suckling kids and lambs. Spanish J Agric Res, 10: 690-700.

Teixeira, A.; Cadavez, V.; Delfa, R. and Bueno, M.S. 2004. Carcass conformation and joints composition of Churra Galega Bragançana and crossbred lambs by Suffolk and Merino Precoce sire breeds. Spanish J Agric Res, 2: 217-226.

Teixeira, A.; Batista, A. S.; Delfa, R. and Cadavez, V. 2005. Lamb meat quality of two breeds with protected origin designation. Influence of breed, sex and live weight. Meat Sci, 71: 530-536.

Teixeira, A.; Jiménez-Badillo, M.R. and Rodrigues, S. 2011 . Effect of sex and carcass weight on carcass traits and meat quality in goat kids of Cabrito Transmontano. Spanish J Agric Res, 9: 753-760.

Teixeira, A.; Sañudo, C.; Olleta, J.L.; Joy, M. y Sanz, A. 2014. Marcas de calidad de carne ovina en la península lbérica. In: Guía práctica de producción ovina en pequeña escala en Iberoamérica. Edited by CYTED, 10/2014. pp. 168-177.

UNE-EN ISO/IEC 17065:2012. Evaluación de la conformidad. Requisitos para organismos que certifican productos, procesos y servicios. (ISO/IEC 17065:2012). 